\title{
Probing spin dynamics from the Mott insulating to the superfluid regime in a dipolar lattice gas
}

\author{
A. de Paz, ${ }^{1,2}$ P. Pedri, ${ }^{1,2}$ A. Sharma, ${ }^{1,3}$ M. Efremov, ${ }^{1,4}$ B. Naylor, ${ }^{2,1}$ O. Gorceix,,${ }^{1,2}$ \\ E. Maréchal, ${ }^{2,1}$ L. Vernac, ${ }^{1,2}$ and B. Laburthe-Tolra ${ }^{2,1}$ \\ ${ }^{1}$ Université Paris 13, Sorbonne Paris Cité, Laboratoire de Physique des Lasers, F-93430 Villetaneuse, France \\ ${ }^{2}$ CNRS, UMR 7538, LPL, F-93430 Villetaneuse, France \\ ${ }^{3}$ Department of Physics and Astronomy, University of Sussex, Brighton BN1 9QH, United Kingdom \\ ${ }^{4}$ Institut fur Quantenphysik, Universität Ulm, Albert-Einstein-Allee 11, D-89081 Ulm, Germany \\ (Received 15 July 2015; revised manuscript received 8 October 2015; published 2 February 2016)
}

\begin{abstract}
We analyze the spin dynamics of an out-of-equilibrium large spin dipolar atomic Bose gas in an optical lattice. We observe a smooth crossover from a complex oscillatory behavior to an exponential behavior throughout the Mott-to-superfluid transition. While both of these regimes are well described by our theoretical models, we provide data in the intermediate regime where dipolar interactions, contact interactions, and superexchange mechanisms compete. In this strongly correlated regime, spin dynamics and transport are coupled, which challenges theoretical models for quantum magnetism.
\end{abstract}

DOI: 10.1103/PhysRevA.93.021603

Dipolar atoms and molecules loaded in optical lattices are a promising platform to study quantum many-body physics [1,2], and, in particular, quantum magnetism [3-8]. In dipolar systems, direct spin-spin interactions are provided by the dipole-dipole interaction (DDI) without relying on a superexchange mechanism [9]. Although magnetization changing collisions associated with the anisotropic character of dipolar interactions may introduce interesting exotic quantum phases [10-13], these off-resonant processes are often negligible. Then, dipolar interactions reduce to the following Hamiltonian,

$$
V_{\mathrm{sc}}=\frac{d^{2}}{r^{3}}\left(S_{1}^{z} S_{2}^{z}-\frac{S_{1}^{+} S_{2}^{-}+S_{1}^{-} S_{2}^{+}}{4}\right)\left[1-3 \cos ^{2}\left(\theta_{1,2}\right)\right],
$$

where $d^{2}=\mu_{0} / 4 \pi\left(g \mu_{B}\right)^{2}\left(\mu_{0}\right.$ being the magnetic permeability of vacuum, $g$ the Landé factor, and $\mu_{B}$ the Bohr magneton), $r$ is the distance between atoms, $\theta_{1,2}$ the angle between the magnetic field and the interatomic axis, and $S_{i}^{ \pm, z}$ are the spin operators acting on atom $i$. This Hamiltonian, known as the secular dipolar Hamiltonian in the context of nuclear magnetic resonance [14], bears strong similarities to the $X X Z$ model of quantum magnetism [9].

Experimental investigations of such spin Hamiltonians have recently started, with dipolar molecules [15], and magnetic [16] and Rydberg [17] atoms, which have raised great interest $[8,10-13,18]$. While these studies have focused on a localized regime where the particles are pinned to a well-defined position, in this Rapid Communication we investigate the case where magnetic atoms are free to move in an optical lattice. Thus spin dynamics and transport are coupled due to an interplay between superexchange mechanisms and dipolar spin exchange. Our experiment provides data in this regime which challenges theoretical descriptions.

We study the spin-exchange dynamics of magnetic chromium ${ }^{52} \mathrm{Cr}$ bosonic atoms loaded in a three-dimensional (3D) optical lattice, across the Mott-to-superfluid transition [19]. We observe, as a function of the lattice depth, a crossover between two distinct behaviors. In the Mott phase, the spin dynamics displays a complex oscillatory behavior, as already studied in Ref. [16]. Although the physics is inherently many body due to strong correlations and the long-range nature of the dipolar interactions, we provide a quantitative interpretation of the oscillations due to intersite DDIs, using a simple model based on perturbation theory. In the superfluid regime, the spin dynamics shows an exponential behavior. Our data are then in good agreement with our numerical simulations based on the Gross-Pitaevskii equation. Dynamics then results from an interplay between contact and dipolar interactions. The regime at an intermediate lattice depth is particularly interesting because superexchange mechanisms also contribute to the spin dynamics. For example, an atom may tunnel into an already occupied site and interact with another atom by spin-dependent contact interactions; this can trigger spin changing collisions [see Fig. 1(b)]. It is extremely challenging to simulate the many-body quantum spin dynamics in this intermediate regime where three exchange mechanisms compete (associated with DDIs, contact interactions, and superexchange). We experimentally find that the oscillations observed in the Mott phase then survive with a reduced amplitude, which constitutes the main result of this work.

Experimental observations. We perform our experiment with a spin- $3{ }^{52} \mathrm{Cr}$ Bose-Einstein condensate, comprising $10^{4}$ atoms loaded into an anisotropic optical lattice [16]. As the lattice depth is spanned, we observe the superfluid-to-Mott transition, at a typical lattice depth of $12 E_{r}$ (where $E_{r}$ is the recoil energy). For our experimental parameters, the system in the Mott phase consists of a core with two atoms per site, surrounded by a shell with one atom per site.

To initiate the spin dynamics, atoms are transferred into the first single-particle excited Zeeman state $m_{s}=-2$ using the tensor light shift of a 427.85-nm light pulse [16]. We then measure, after a variable hold time $t$, the spin populations by means of a Stern-Gerlach procedure. After a ramp of the tensor light shift, typically $45 \pm 5 \%$ of the atoms are measured in the state $m_{s}=-2$ at $t=0$. The uncertainty includes a systematic effect due to nonvanishing spin dynamics after the atoms are released from the lattice. This $\approx 10 \%$ effect is most pronounced at low lattice depths. A typical result of spin dynamics is plotted in Fig. 1(c). Only the $m_{s}=-3,-2$, -1 , and 0 spin components are significantly populated as the system evolves. The populations display a rather complex 

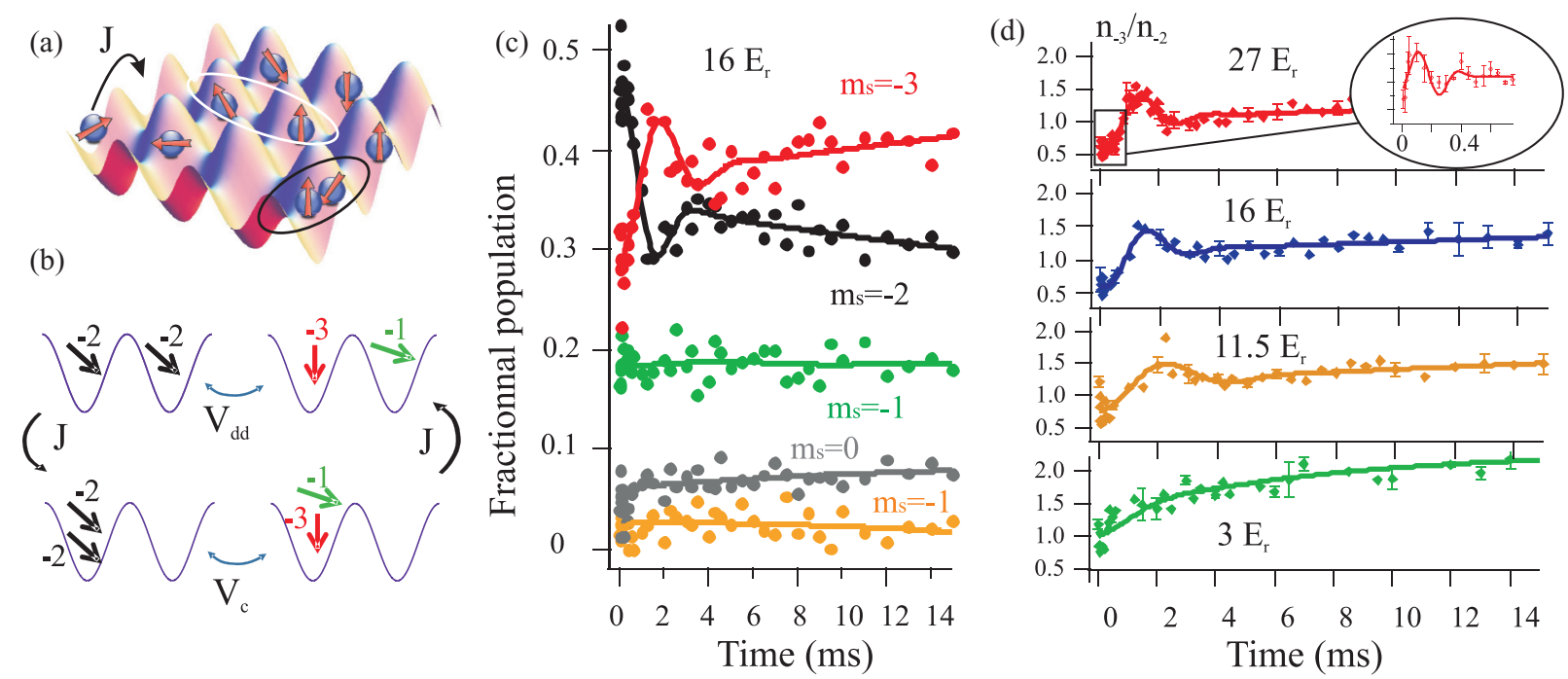

FIG. 1. (a) Simple representation of the system close to the Mott-to-superfluid transition. Atoms interact both due to intersite (white ellipse) and on-site (black ellipse) interactions. (b) Sketch illustrating the competition between exchange due to DDI $\left(V_{d d}\right)$ and tunneling $(J)$ assisted spin exchange due to contact interactions $\left(V_{c}\right)$. (c) Measurement of the spin components as a function of time for $16 E_{r}$. (d) Time evolution of observable $n_{-3} / n_{-2}$ for four different lattice depths $\left(27 E_{r}, 16 E_{r}, 11.5 E_{r}\right.$, and $3 E_{r}$, from top to bottom). Lines are guides for the eye resulting from fits.

behavior as a function of time. In order to simplify the discussion, we focus our attention on the observable given by the ratio $n_{-3} / n_{-2}$ of the $m_{s}=-3$ and $m_{s}=-2$ populations, since they are the most populated components.

We have plotted in Fig. 1(d) the typical results corresponding to four different lattice depths $\left(27 E_{r}, 16 E_{r}, 11.5 E_{r}\right.$, and $3 E_{r}$ ), showing quite different spin dynamics (see also the extended data in Ref. [20]). In the Mott phase at short times we observe $(<0.5 \mathrm{~ms}$, see the inset) a strongly damped oscillation, and then at longer times a second oscillation. In the superfluid phase the spin dynamics is better described by an exponential. All these features are present in the data from $3 E_{r}$ to $27 E_{r}$. We use the following empirical function, $f(t)=f_{0}+A \cos (\omega t) \exp \left[-\left(t / t_{0}\right)^{2}\right]+B \exp \left(-t / t_{1}\right)$, to fit to our experimental data for $t>0.5 \mathrm{~ms}$. We plot in Fig. 2(a) the amplitudes of the exponential $B$ and of the slow oscillation $A$, and in Fig. 2(b) the frequencies of the slow oscillations $(\omega)$. The frequency $\Omega$ of the fast oscillation is deduced from a sinusoidal fit to independent data taken specifically to study the short-time $t<0.5 \mathrm{~ms}$ behavior. When reducing the lattice depth, we observe that both oscillation frequencies $\omega$ and $\Omega$ decrease and they become closer to each other. Oscillations do survive at lattice depths slightly below the Mott-to-superfluid transition. However, for very shallow lattice depths, the oscillations at low frequency are barely visible and the spin dynamics is mostly exponential.

For most of the data shown in Fig. 2 the magnetization is constant [see the inset of Fig. 2(a)]. The stability of the magnetization for large lattice depths indicates that dipolar relaxation is suppressed, which arises because the energy released in a dipolar relaxation event (the Larmor energy $\approx 30 \mathrm{kHz}$ ) does not match the band excitation $(>50 \mathrm{kHz})[21]$. At small lattice depths, dipolar relaxation is not completely suppressed (as the first excited band has an energy close to the Larmor energy). However, this effect remains rather small, and is neglected in the theoretical analysis presented below.

Theoretical interpretation at low lattice depths. To account for the dynamics at the lowest lattice depths, the GrossPitaevskii equation can be safely used because the gas is in the condensate phase. We performed a numerical simulation up to a lattice depth of $7 E_{r}$ in order to describe the observed data. The interaction term takes into account short-range contact interactions and nonlocal DDIs [20]. Concerning DDIs, we only include the spin-conserving terms (see above).

Simulations display a complex behavior (see Fig. 3 for $7 E_{r}$ ). The general trend of the experimental spin dynamics, showing a slow drift with $\mathrm{a} \approx 14 \mathrm{~ms}$ characteristic $1 / e$ time, is well reproduced by the simulation. By fitting both curves (experimental and numerical) by an exponential, a good agreement for the amplitude is found [see Fig. 2(a)].

In addition, a Fourier analysis of the numerical results (up to $15 \mathrm{~ms}$ ) displays a lowest resolved frequency in good agreement with the experimentally observed frequency $\Omega$ at a lattice depth of $3 E_{r}$ and $7 E_{r}$ [see Fig. 2(b)]. Higher frequencies are not resolved in our experiment due to time resolution. The strong damping observed in the experiment is not reproduced by the zero-temperature simulation. For very low lattice depths (below $3 E_{r}$ ), simulations show that both contact and dipole interactions contribute to the spin dynamics. In particular, if we set the DDI to zero, we numerically observe a spin dynamics frequency which is roughly twice faster. This illustrates the interplay between dipolar and contact interactions in the superfluid regime.

We also investigate numerically the spatial dependence of the dynamics in Fig. 3(b), which shows a cut of the density of the atoms in the $m_{s}=-2$ Zeeman state in the absence of an optical lattice, for three evolution times. Similar results are obtained at small lattice depths. The dynamics is inhomogeneous, due to a nontrivial interplay between 

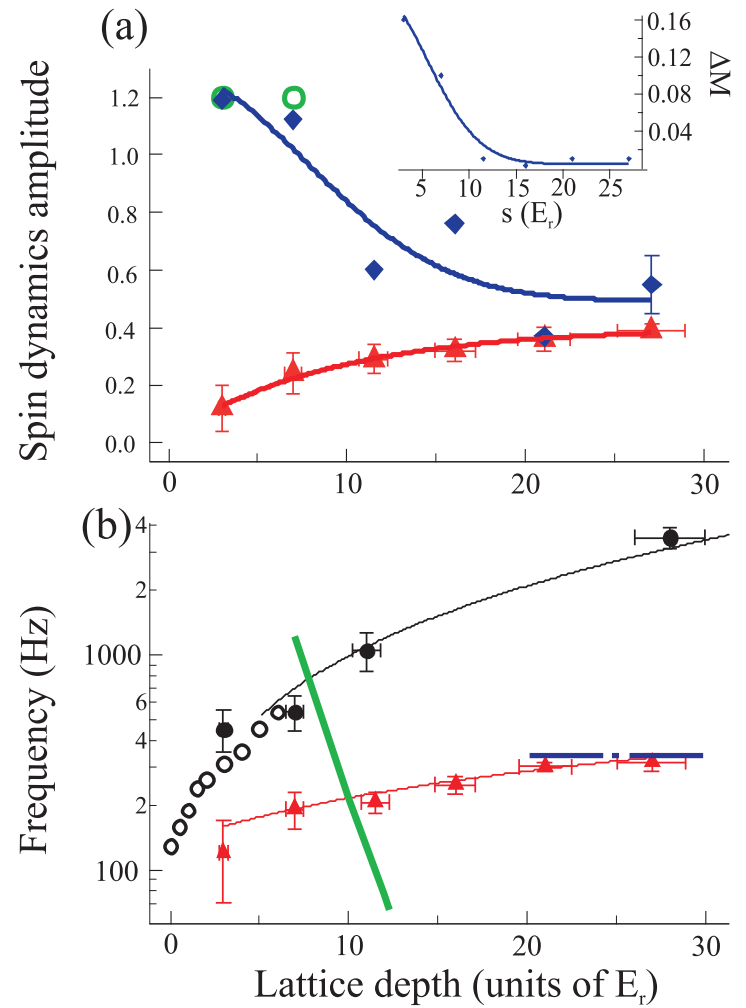

FIG. 2. Spin dynamics amplitudes and frequencies as a function of the lattice depth. (a) Amplitude of the exponential dynamics (blue diamonds) and slow oscillation (red triangles). Green circles are results of numerical simulations. Inset: Variation of the magnetization over $20 \mathrm{~ms}$. Solid lines are guides to the eye. (b) Frequency of fast (black points) and slow (red triangles) oscillations. The top black solid line corresponds to spin-exchange frequency associated with intrasite contact interactions, while the black open circles correspond to a numerical simulation of the Gross-Pitaevskii equation. The bottom red curve is a guide to the eye. The blue dotted-dashed line shows the prediction in the Mott regime (see text). The frequency of the superexchange process is given by the green solid line. Error bars in frequency and amplitude result from the statistical uncertainty in the fits.

the contact and dipolar interactions. While spin-exchange interactions due to contact interactions are larger in the center of the cloud, due to a higher density, dipolar interactions are stronger in the outlying areas [22]. The observed dynamics is faster in the center, which illustrates the dominant role of contact interactions at low lattice depths.

Theoretical interpretation at large lattice depths. We now turn to our theoretical analysis at large lattice depths, where the system is not superfluid and one should go beyond the Gross-Pitaevskii mean-field description. We focus on the frequencies of the two oscillations observed in the Mott phase. We stress that for the largest lattice depth, the experiment is performed in a regime where tunneling is practically absent, and superexchange interactions are exponentially reduced, as shown in Fig. 2(b).

As shown in Ref. [16], we interpret the upper frequency as a result of the intrasite spin-exchange dynamics $(|-2,-2\rangle \rightarrow$ $\frac{1}{\sqrt{2}}(|-3,-1\rangle+|-1,-3\rangle)$ arising from spin-dependent contact interactions in doubly occupied sites. The observed $\mathrm{n}_{-3} / \mathrm{n}_{-2}$

(a)
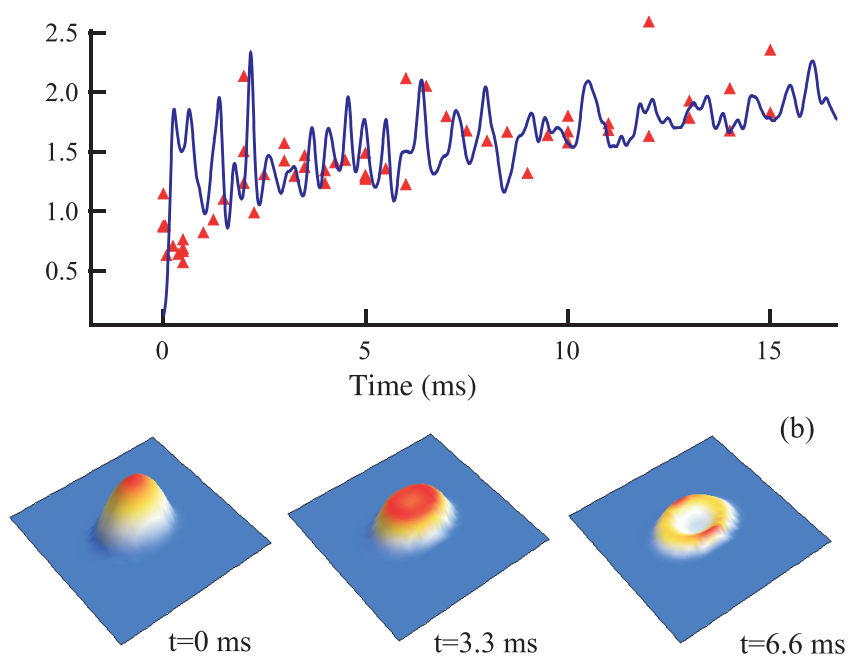

FIG. 3. Results of numerical simulations using the GrossPitaevskii equation. (a) Spin dynamics for the deepest lattice depth that could be simulated ( $7 E_{r}$, solid line). The red triangles are experimental data. (b) Spatial analysis of spin dynamics, showing a cut of the density of the atoms in the $m_{s}=-2$ state along a horizontal plane $z=0$ (with no lattice on).

frequency is in good agreement with the theoretical frequency $\frac{4 \pi \hbar^{2}}{2 \sqrt{2} m}\left(a_{6}-a_{4}\right) n_{0}$ [see the black solid line in Fig. 2(b)]. Here, $n_{0}$ is the peak density in a doubly occupied lattice site, $m$ the atom mass; $a_{6}$ and $a_{4}$ are the scattering lengths of the $S=6$ and $S=4$ molecular channels, respectively [23,24].

As for the lower frequency, we associate it to the nonlocal DDI between doublons, which was also described in Ref. [16]. Although a realistic simulation of our results based on calculating the exact dynamics associated with the full many-body Hamiltonian is out of reach of the present computational techniques, we propose a perturbative model which quantitatively accounts for the many-body interactions between doublons due to intersite DDIs. We first assume that, after the damping of the fast oscillations, the ensemble of doublons is in a statistical mixture of $\left|S=6, m_{s}=4\right\rangle$ and $\left|S=4, m_{s}=4\right\rangle$. We then describe the spin dynamics due to the intersite DDI between doubly occupied sites by the following model, inspired by Refs. [14,25]. We calculate the time evolution of the population $N_{-2}$ in the state $m_{s}=-2$ using perturbation theory in the Heisenberg picture. The many-body Hamiltonian takes into account the interaction of one doubly occupied lattice site $i$ with all its neighbors $j$ by a pairwise DDI. Using a Taylor expansion, the formal expression of the population reads $N_{-2}(t)=\sum_{n=0}^{+\infty} M_{n} t^{n}$. While $M_{1}$ and $M_{3}$ vanish, the second moment $M_{2}$ describing the spin dynamics up to the second order of perturbation reads

$$
M_{2}=-\sum_{j \neq i} V_{d d}\left(r_{i, j}\right)^{2} / \hbar^{2},
$$

where $V_{d d}\left(r_{i, j}\right)$ is the dipolar spin-exchange matrix element between sites $i$ and $j$ [20].

From $M_{2}$, we first extract an estimate of the spin oscillation frequency $v: \cos ^{2}(\pi v t) \approx 1-M_{2} t^{2}$. However, as shown in 
Ref. [14], the higher-order terms are expected to lead to a reduction of the spin dynamics amplitude and a decrease of the quasiperiod typically by a factor of 2 . We indeed recover these features by taking into account the moment up to the fourth order $M_{4}$ (following the calculation in Ref. [14]). We apply this perturbative approach up to fourth order to the case of an assembly of doublons in a state $\left|S=6, m_{s}=4\right\rangle$, for which intersite spin-exchange interactions are strongest.

The result is displayed in Fig. 2(b), and shows good agreement with the observed frequencies, which illustrates the relevance of our model in the deep Mott regime. The observed reduction of the frequency at lower lattice depths may be a consequence of the reduction of the doubly occupied site Mott plateau, leading to either stronger border effects or more defects. The reduction of the frequency in the presence of holes is also an expected feature [14]. The damping (also observed in Ref. [14]) is not reproduced by our theoretical model, which can only account for dynamics at short times. In general, it should be stressed that the many-body system which we study here is extremely challenging for nonperturbative many-body simulations, due to the large spin and the immense Hilbert space which has to be taken into account.

In this Rapid Communication we have explored the spin dynamics of chromium atoms loaded in an optical lattice as a function of the lattice depth. Superfluid and Mott regimes lead to markedly different features. Experimentally, the spin dynamics evolves smoothly when we vary the lattice depth between these two extreme regimes. However, our analysis shows that the impact of DDIs on spin dynamics is drastically different in the two regimes: Whereas in the weakly interacting regime, the dipolar interactions are described by a mean field associated with a geometrical average, in the strongly correlated regime, dipole-dipole couplings from one site to the other sites are added quadratically. One important consequence is that spin dynamics mediated by dipolar interactions should be a border effect in the mean-field regime, but a bulk effect in the strongly correlated gas [20]. Space-resolved measurements could therefore be a very interesting way to discriminate both regimes.

Whereas the two extreme regimes of shallow and deep lattice depths are qualitatively well described by our theoretical models, the main unusual aspect of this work is the experimental study of the regime close to the Mott-to-superfluid transition. In this case, exchange processes due to dipolar interactions, spin exchange due to contact interactions, and superexchange interactions may all contribute to the dynamics on the same footing. Our experiment provides insights into the coupled out-of-equilibrium magnetic and transport properties of such a strongly correlated gas, which challenges theoretical descriptions. Our work thus adds to the study of the magnetic properties of an exotic quantum many-body system made of large spin dipolar particles.

\section{ACKNOWLEDGMENTS}

This work was supported by Conseil Régional d'Ile-de-France under DIM Nano-K/IFRAF, Ministère de l'Enseignement Supérieur et de la Recherche within CPER Contract, and by Université Sorbonne Paris Cité (USPC). P.P. acknowledges funding by the Indo-French Centre for the Promotion of Advanced Research-CEFIPRA. We thank Martin Robert-deSaint-Vincent for his critical reading of the manuscript.
[1] M. A. Baranov, M. Dalmonte, G. Pupillo, and P. Zoller, Chem. Rev. 112, 5012 (2012); Cold Molecules: Theory, Experiment, Applications, edited by R. V. Krems, W. C. Stwalley, and B. Friedrich (Taylor and Francis/CRC, Boca Raton, FL, 2009); T. Lahaye, C. Menotti, L. Santos, M. Lewenstein, and T. Pfau, Rep. Prog. Phys. 72, 126401 (2009); M. Baranov, Phys. Rep. 464, 71 (2008).

[2] S. Baier, M. J. Mark, D. Petter, K. Aikawa, L. Chomaz, Z. Cai, M. Baranov, P. Zoller, and F. Ferlaino, arXiv:1507.03500.

[3] R. Barnett, D. Petrov, M. Lukin, and E. Demler, Phys. Rev. Lett. 96, 190401 (2006).

[4] A. Micheli, G. K. Brennen, and P. Zoller, Nat. Phys. 2, 341 (2006).

[5] A. V. Gorshkov et al., Phys. Rev. Lett. 107, 115301 (2011).

[6] D. Peter, S. Muller, S. Wessel, and H. P. Buchler, Phys. Rev. Lett. 109, 025303 (2012).

[7] M. L. Wall, K. Maeda, and L. D. Carr, Ann. Phys. 525, 845 (2013).

[8] K. R. A. Hazzard, M. van den Worm, M. Foss-Feig, S. R. Manmana, E. G. Dalla Torre, T. Pfau, M. Kastner, and A. M. Rey, Phys. Rev. A 90, 063622 (2014).

[9] A. Auerbach, Interacting Electrons and Quantum Magnetism (Springer, New York, 1994).

[10] D. Peter, N. Y. Yao, N. Lang, S. D. Huber, M. D. Lukin, and H. P. Buchler, Phys. Rev. A 91, 053617 (2015).
[11] M. L. Wall, K. Maeda, and L. D. Carr, New J. Phys. 17, 025001 (2015).

[12] A. W. Glaetzle, M. Dalmonte, R. Nath, C. Gross, I. Bloch, and P. Zoller, Phys. Rev. Lett. 114, 173002 (2015).

[13] S. V. Syzranov, M. L. Wall, V. Gurarie, and A. M. Rey, Nat. Commun. 5, 5391 (2014).

[14] A. Abragam, Principles of Nuclear Magnetism (Clarendon, Oxford, UK, 1983)

[15] B. Yan, S. A. Moses, B. Gadway, J. P. Covey, K. R. A. Hazzard, A. M. Rey, D. S. Jin, and J. Ye, Nature (London) 501, 521 (2013).

[16] A. de Paz, A. Sharma, A. Chotia, E. Maréchal, J. H. Huckans, P. Pedri, L. Santos, O. Gorceix, L. Vernac, and B. Laburthe-Tolra, Phys. Rev. Lett. 111, 185305 (2013).

[17] D. Barredo, H. Labuhn, S. Ravets, T. Lahaye, A. Browaeys, and C. S. Adams, Phys. Rev. Lett. 114, 113002 (2015).

[18] See, for example, N. Y. Yao, C. R. Laumann, S. Gopalakrishnan, M. Knap, M. Mueller, E. A. Demler, and M. D. Lukin, Phys. Rev. Lett. 113, 243002 (2014); J. Schachenmayer, A. Pikovski, and A. M. Rey, Phys. Rev. X 5, 011022 (2015); R. M. W. van Bijnen and T. Pohl, Phys. Rev. Lett. 114, 243002 (2015); L. Barbiero, C. Menotti, A. Recati, and L. Santos, Phys. Rev. B 92, 180406 (2015); Z.-X. Gong, M. F. Maghrebi, A. Hu, M. L. Wall, M. Foss-Feig, and A. V. Gorshkov, ibid. 93, 041102 (2016); O. Dutta, M. Gajda, P. Hauke, M. Lewenstein, D.-S. Lühmann, B. 
A. Malomed, T. Sowinski, and J. Zakrzewski, Rep. Prog. Phys. 78, 066001 (2015).

[19] M. Greiner, O. Mandel, T. Esslinger, T. W. Hansch, and I. Bloch, Nature (London) 415, 39 (2002).

[20] See Supplemental Material at http://link.aps.org/supplemental/ 10.1103/PhysRevA.93.021603 for extended data, details on numerical simulations, and discussions on how the mean-field and the strongly correlated regime differ.

[21] A. de Paz, A. Chotia, E. Maréchal, P. Pedri, L. Vernac, O. Gorceix, and B. Laburthe-Tolra, Phys. Rev. A 87, 051609(R) (2013).
[22] D. H. J. O’Dell, S. Giovanazzi, and C. Eberlein, Phys. Rev. Lett. 92, 250401 (2004).

[23] J. Werner, A. Griesmaier, S. Hensler, J. Stuhler, T. Pfau, A. Simoni, and E. Tiesinga, Phys. Rev. Lett. 94, 183201 (2005).

[24] B. Pasquiou, G. Bismut, Q. Beaufils, A. Crubellier, E. Maréchal, P. Pedri, L. Vernac, O. Gorceix, and B. Laburthe-Tolra, Phys. Rev. A 81, 042716 (2010).

[25] R. C. Brown, R. Wyllie, S. B. Koller, E. A. Goldschmidt, M. Foss-Feig, and J. V. Porto, Science 348, 540 (2015). 